\title{
Nanoparticle size, shape, and concentration measurement at once - two VAMAS pre- standardization projects ready to start
}

\author{
Vasile-Dan Hodoroaba ${ }^{1}$, Christoph Hörenz ${ }^{2}$, Francesco Pellegrino ${ }^{3}$, Valter Maurino ${ }^{3}$, Bénédicte Durand ${ }^{4}$ and \\ Olivier Taché ${ }^{4}$ \\ ${ }^{1}$ Federal Institute for Materials Testing and Research (BAM), Division 6.1 Surface Analysis and Interfacial \\ Chemistry, Berlin, Berlin, Germany, ${ }^{2}$ Federal Institute for Materials Research and Testing (BAM), Germany, \\ Berlin, Berlin, Germany, ${ }^{3}$ Dipartimento di Chimica and Centro Interdipartimentale NIS, Università di Torino, \\ Italy, United States, ${ }^{4}$ Commissariat à l'énergie atomique et aux énergies alternatives (CEA), France, United \\ States
}

The research and development of nanoparticulate materials has been emerging for decades. Today they provide clever solutions and promising prospects for current and possibly for future problems. However, the traceable morphological and chemical characterization of nanomaterials remains challenging with respect to the various possible sizes, size distributions, shapes, and concentrations of real-world nanoparticles (NPs). A cause and, at the same time, a consequence of this situation is the lack of standard measurement procedures accompanied by a reduced number of available certified reference materials.

In order to address this problem, the project 'nPSize - Improved traceability chain of nanoparticle size measurements' was initiated in the framework of the EU-EMPIR initiative. The development of traceable measurement procedures for size, shape, and concentration covers a central part of the project including only measurement techniques able to provide traceable nanoparticle sizes and number concentration: Electron Microscopy (SEM, STEM-in-SEM and TEM), Atomic Force Microscopy, Small Angle X-ray Scattering (SAXS) and single-particle ICP-MS. A unique set of NPs such as spherical, cubical, bipyramidal, platelets and elongated particles, with monodisperse, polydisperse (including bimodal) size distributions was developed within the project [1,2]. The range of materials comprises $\mathrm{TiO}_{2}, \mathrm{SiO}_{2}$, and gold.

A case study on the analysis of the size and shape distribution of $\mathrm{TiO}_{2}$ bipyramidal NPs by TEM was included in the recently published ISO standard ISO 21363 [3]. It was agreed to organize first an inter-laboratory study with nanoparticles already prepared according to the same procedure on a TEM grid [3], and, at a later stage to carry out a second inter-lab comparison with the nanoparticles distributed to the participants as a liquid suspension. Protocols for uniform nanoparticle deposition on suited supports developed and optimized within nPSize (see Figure 1) are also prepared to be distributed. For this, we have chosen the VAMAS platform (www.vamas.org) which offers an excellent international infrastructure of laboratories with high competence in nanoparticle measurement. The VAMAS technical working area dedicated to nanoparticle measurement is TWA 34 'Nanoparticle populations'. For this type of nanoparticles, the size and shape distributions are the primary parameters to be reported. Due to the good deposition protocols developed, an automated image analysis is enabled (in contrast to the manual analysis of irregular $\mathrm{TiO}_{2}$ nanoparticles [4]). For the measurement of the size distribution with SEM, AFM and SAXS, standards are available or in progress [5-7]. SAXS and spICP-MS are expected to also provide the number concentration.

In parallel with the $\mathrm{TiO}_{2}$ nanoparticle exercise, two spherical $\mathrm{SiO}_{2}$ nanoparticle samples with bi-modal size distributions (nominal relative number concentrations of 1:1 and 10:1) are prepared for a second VAMAS inter-laboratory comparison (see Figure 1). Here, the nanoparticle concentration is the primary parameter to be measured. For the imaging methods it is targeted to measure the relative nanoparticle concentrations (relative populations of the two modes). For the absolute nanoparticle concentration to be measured by imaging methods it is necessary to control the volume of the liquid suspension deposited on the substrate and to obtain such a homogeneous nanoparticle deposition on the substrate which allows to count (or extrapolate) all the deposited particles. 
Both projects are short before start, more information including the participation call will be published soon on the VAMAS webpage (http://www.vamas.org/twa34). 200 ampoules of each type of suspension, of $2 \mathrm{~mL}$ for $\mathrm{TiO}_{2}$ bipyramids and of $1 \mathrm{~mL}$ for the silica bi-modal samples, are available. Based on the results collected from the two inter-laboratory comparisons, these types of nanoparticles are planned to be certified by BAM with respect to their size and shape distribution and concentration and, hence, to improve the poor offer of certified nanoparticles available worldwide [8].

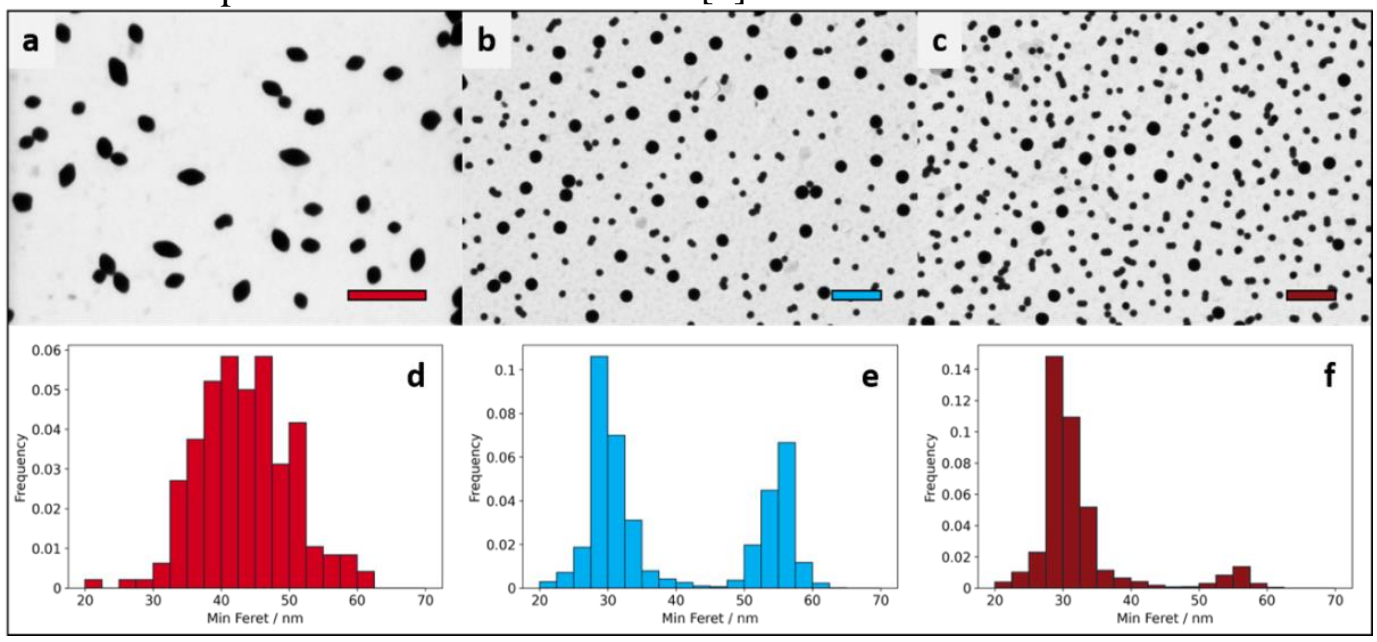

Figure 1. STEM-in-SEM micrographs (a-c) of bipyramidal $\mathrm{TiO}_{2}$ nanoparticles (a), bi-modal $\mathrm{SiO}_{2}$ (nominal 1:1 concentration ratio (b), and bi-modal $\mathrm{SiO}_{2}$ (10:1 nominal concentration ratio (c)), and their corresponding particle size distributions (d-f). Scale bars represent $200 \mathrm{~nm}$. Note that the histograms were obtained from a series of micrographs.

\section{References}

[1] C Hörenz et al, Microsc. Microanal. 26 (2020), p. 2282.

[2] U Mansfeld et al, Microsc. Microanal. 25 (Suppl 2) (2019), p. 2328.

[3] ISO 21363:2020 Nanotechnologies - Measurements of particle size and shape distributions by transmission electron microscopy. Geneva, Switzerland: ISO.

[4] E A Grulke et al, Adv. Powder Tech. 28 (2017), p. 1647.

[5] ISO/PRF 19749 Nanotechnologies - Measurements of particle size and shape distributions by scanning electron microscopy. Geneva, Switzerland: ISO.

[6] ISO 17867:2020 Particle size analysis - Small angle X-ray scattering (SAXS). Geneva, Switzerland: ISO. [7] VAMAS/TWA2, Project A24, Guidelines for shape and size analysis of nano-particles by AFM (www.vamas.org/twa2).

[8] This project 17NRM04 nPSize has received funding from the EMPIR programme co-financed by the Participating States and from the European Union's Horizon 2020 research and innovation programme. 